\title{
Risk of Intussusception After Rotavirus Vaccination
}

A Systematic Literature Review and Meta-Analysis

Judith Koch, Thomas Harder, Rüdiger von Kries, Ole Wichmann

\section{SUMMARY}

Background: In 2013, the German Standing Committee on Vaccination (Ständige Impfkommission, STIKO) recommended rotavirus (RV) vaccination for all infants while stating that this mildly increased the risk of intussusception, a potentially life-threatening event. Since this recommendation was issued, multiple observational studies on this topic designed as self-controlled case series (SCCS) have been published. The SCCS design is particularly suitable for the study of rare adverse effects of medications.

Methods: We systematically searched the literature for SCCS studies on the risk of intussusception after RV vaccination. Relative risks (RR) corresponding to different doses of vaccine were summarized in a meta-analysis, and attributable risks (AR) were calculated.

Results: Of the 16 initially identified studies, 10 with a predominantly low risk of bias were considered in the analysis. The RR for intussusception was 5.71 (95\% confidence interval [4.50; 7.25]) 1-7 days after the first dose, 1.69 [1.33; $2.14]$ after the second, and $1.14[0.75 ; 1.74]$ after the third. The AR for children of the age at which RV vaccination is recommended was $1.7[1.1 ; 2.7]$ additional intussusceptions per 100000 vaccinated children after the first dose and $0.25[0.16 ; 0.40]$ after the second. If $>3$-month-old infants are vaccinated, the AR is higher: 5.6 [4.3; 7.2] per 100000 after the first dose and $0.81[0.63 ; 1.06]$ per 100000 after the second.

Conclusion: RV vaccination is associated with a markedly elevated RR and a mildly elevated AR for intussusception 1-7 days after the first dose. Physicians should begin the series of vaccinations at age 6-12 weeks, as recommended by the STIKO, because the risk of intussusception is higher afterward. Current health insurance company claim data indicate that $11.2 \%$ of infants are still receiving the first dose of the vaccine at ages above 3 months. The parents of vaccinated children should be informed about the possible signs of intussusception (colicky pain, bilious vomiting, and red "currant jelly" stool).

\section{$\rightarrow$ Cite this as:}

Koch J, Harder T, von Kries R, Wichmann 0: The risk of intussusception after rotavirus vaccination-a systematic literature review and meta-analysis. Dtsch Arztebl Int 2017; 114: 255-62. DOI: 10.3238/arztebl.2017.0255
Immunization Unit, Robert Koch Institute Berlin, Robert Koch Institute Berlin: Dr. med. Koch, Dr. med. Harder, PD Dr. med. Wichmann

Institute of Social Paediatrics and Adolescent Medicine, Ludwig-Maximilians-Universität München: Prof. Dr. med. von Kries
D otaviruses (RV) are the commonest cause of diarrhea in infants and young children (1). The main symptoms of rotavirus-related gastroenteritis (RVGE) are watery, non-bloody diarrhea with vomiting and fever lasting from 4 to 7 days. According to international estimates, 453000 deaths in children under the age of 5 every year worldwide are due to $\mathrm{RV}$ infection (2). In Germany, before vaccination was introduced, RVGE was the commonest notifiable disease in under 5-year-olds; the highest annual incidence was seen in children under the age of 2 (1850 / 100 000) (3). Because of fluid and electrolyte loss, inpatient admission to hospital for replacement therapy is often required; in children under the age of 5 , the hospital admission rate is about $50 \%$ of the cases reported under the Notifiable Diseases Act (3).

Since June 2006, two second-generation RV vaccines (RV1 [monovalent] and RV5 [pentavalent]) have been available in Germany. These are oral live vaccines used for active immunization of infants to prevent severe RV disease. Analysis of data received from the associations of statutory health insurance physicians ( $\mathrm{KV}$, Kassenärztliche Vereinigungen) in the course of vaccination monitoring indicated a $\mathrm{RV}$ vaccination rate of over $70 \%$ in 2015 , showing a rising trend in comparison to the previous year (Thorsten Rieck, Robert Koch Institute, personal communication) (4).

Depending on the vaccine used, RV vaccination consists of two (RV1) or three doses (RV5) given to infants at 4-week intervals starting at the age of 6 weeks. The RV1 series of vaccinations should preferably be administered before the age of 16 weeks, and, according to the product information, must be completed by the age of 24 weeks (5). The RV5 vaccination series should be started no later than in the $11^{\text {th }}$ week of life, should preferably be completed by the end of the $20^{\text {th }}$ or $22^{\text {nd }}$ week, and must be completed before the end of the $32^{\text {nd }}$ week of life at the latest (6).

Intussusception is a rare but potentially life-threatening event and is the commonest cause of ileus during infancy. Most intussusceptions are idiopathic, but increased gut motility during a viral infection is also believed to be a cause (7). Data on the background incidence of intussusception in Germany from the period before the RV vaccination era are available. The annual 
incidence in children below the age of 1 year in Germany is estimated to be $61.7 / 100000$ (95\% confidence interval: $[54.5 ; 70.1])$. The incidence during the first year of life varies greatly and rises sharply with age: from 19.2 / $100000[12.5 ; 30.4]$ during the first 3 months of life to $98.5 / 100000[80.9 ; 120.6]$ at the age of 6-8 months (8).

Because a first generation RV vaccine licensed in the USA in 1998 was associated with a 37 -fold increased risk of intussusception and was withdrawn from the market 9 months after licensing, intussusception risk was studied in detail during the safety assessment of the second generation of RV vaccines $(9,10)$. None of the extensive registrational trials of the two vaccines currently on the market showed an association with the occurrence of intussusception $(11,12)$. However, these studies were not designed to capture events that occurred with a probability of less than $1 /$ 50 000. The German Standing Committee on Vaccination (STIKO, Ständige Impfkommission) published its recommendation that infants should receive RV vaccination in August 2013. Based on data from observational studies available at that time, the risk was estimated to be 1 to 2 additional intussusceptions per 100000 vaccinated infants, irrespective of which vaccine was used (13-22). STIKO states explicitly that the vaccination is associated with a slightly increased risk of intussusception during the first week after the first dose of vaccine. In view of the large number of RV-related hospital admissions of young children, however, the benefit of vaccination outweighs the risk. Since the available data suggested that the risk of intussusception increased with the age at vaccination of the infant, STIKO recommends that the vaccination series should be started early and completed in good time.

Since the STIKO recommendation came out, more observational studies have been published, particularly studies using the "self-controlled case series" (SCCS) design, which is less prone to bias error (eBox 1). Our aim was to test the estimated intussusception risk on which the recommendation was based using the new data from the SCCS studies, taking account of variations in vaccination time points and risk intervals. To this end, we carried out a systematic review to answer the following primary questions:

- How high (compared with the situation without vaccination) is the relative risk (RR) for intussusception after RV vaccination?

- Is the RR after the first dose of vaccine different from the RR after the second or third dose of vaccine?

- How high is the attributable risk, i.e., the risk that is attributable to the vaccination? This is the number that allows the absolute increase in risk caused by the vaccination to be quantified.

\section{Methods}

\section{Literature search}

The systematic literature search was carried out in accordance with the requirements of the PRISMA (Pre- ferred Reporting Items for Systematic Reviews and Meta-Analyses) statement (23). The MEDLINE, EMBASE, Cochrane Central Register of Controlled Trials, SciSearch, GLOBAL Health, and BIOSIS Previews databases were all searched (last search date: 4 July 2016). The PRISMA flow chart is shown in eFigure 1 and the search strategy in eBox 2 .

\section{Selection of studies}

The inclusion criteria were defined before the start of the study on the basis of the PICO framework (patient group, intervention, control group, outcome):

- Patient group: Infants up to 1 year of age

- Intervention: Vaccination with a licensed RV vaccine

- Control group: Time period outside the risk period

- Outcome: Intussusception according to the criteria of the Brighton Collaboration (24).

Only studies with a SCCS design were included in the systematic review; this design was developed for the investigation of rare events (details of the study design are given in eBox 1) (25).

\section{Calculation of attributable risk}

Based on the age-specific annual background incidence of intussusceptions per 100000 infants in Germany (8), the number of intussusceptions that would normally occur in a birth cohort (in this case 2014) within the 7 -day risk period was calculated. The calculations were carried out for 1 week of life at the age of $<3$ months and 1 week at the age of $\geq 3$ months. This number was multiplied by the calculated RR for the respective dose of vaccine. The risk attributable to the vaccine was calculated by subtracting from this value the number of background cases occurring per week in the birth cohort. The attributable risk was calculated for various vaccination periods after the first and second doses of vaccine.

\section{Results}

The search produced 25 hits; one additional relevant study was identified during screening of the full publications and reviews. Title and abstract screening resulted in the identification of 16 potentially relevant publications. Ten of these 16 studies met the inclusion criteria for the meta-analysis (Table 1) (18, 26-34). The publications not included in the full text screening are listed as references e2-e7. In the case of the study by Patel et al. (18), only data from Mexico were included; the results from Brazil were ignored, because in Brazil the oral polio vaccine (OPV) was given in parallel with the first dose of RV vaccine. Administration of these two live vaccines in parallel appears to reduce the immunogenicity of the RV vaccine and therefore also to mask the possible intussusception risk (35).

\section{Study characteristics}

Of the 10 studies included in the review, 7 provided data on the risk of intussusception after RV1 vaccination (18, $26,29,30,32-34)$ and 4 on the risk after RV5 


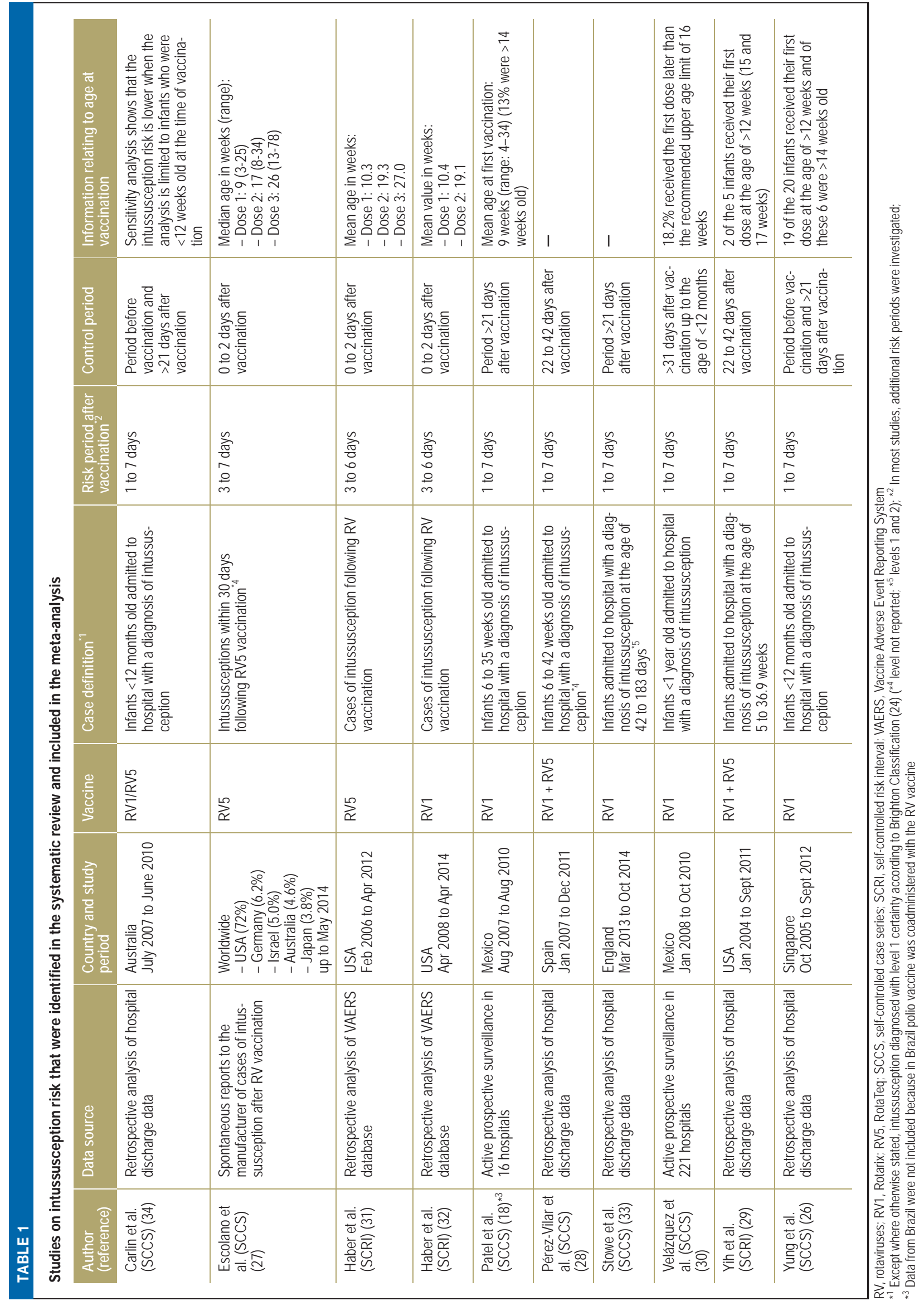


FIGURE 1

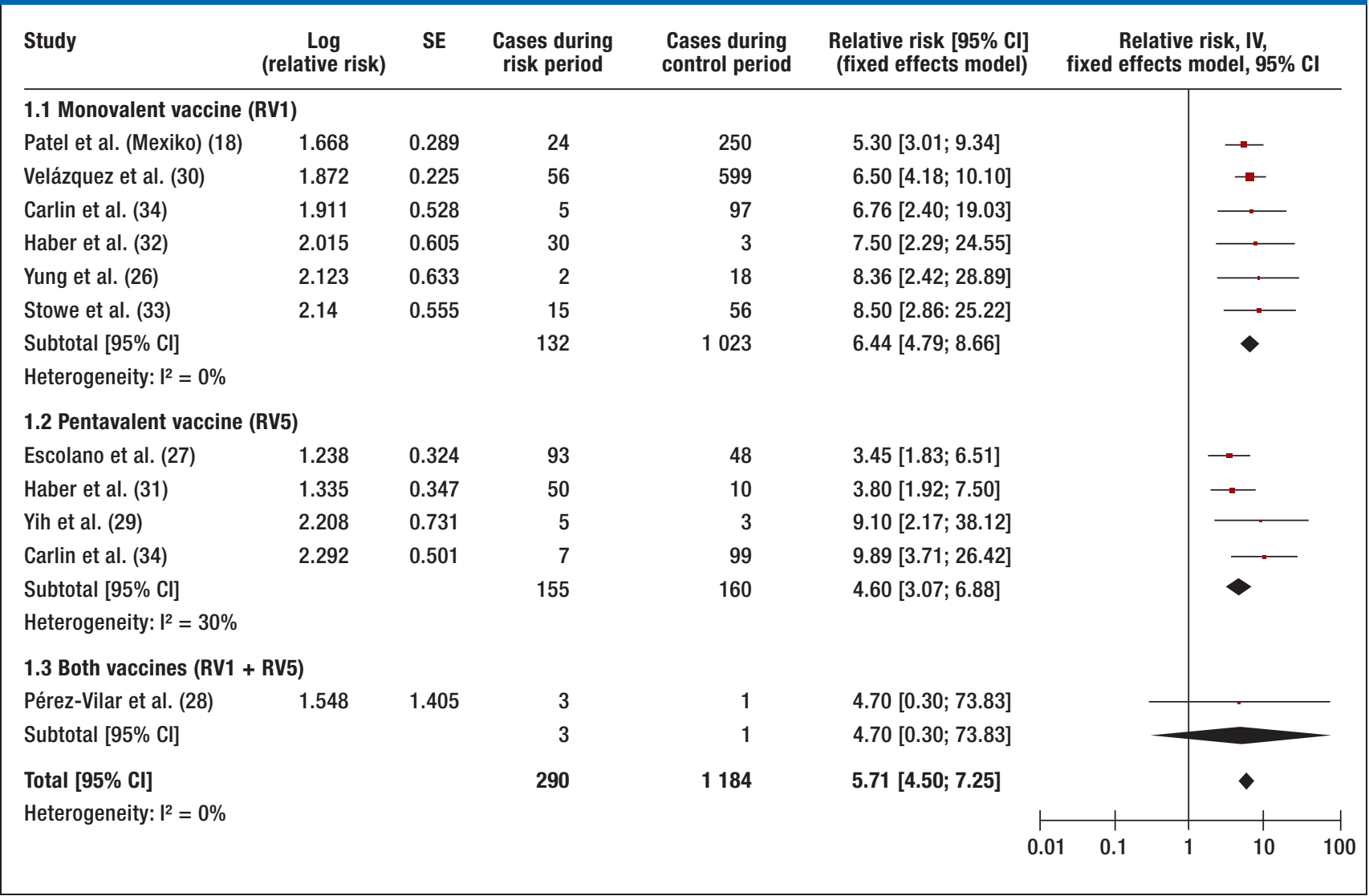

Risk of intussusception within 7 days following the first dose of vaccine

$95 \% \mathrm{Cl}, 95 \%$ confidence interval; IV, inverse variance; SE, standard error

vaccination $(27,29,31,34)$. One study reported only the results for both vaccines together (28). In the RV1 studies the risk incidences were calculated for the risk periods after the first and second doses of vaccine, and in the RV5 studies for the periods after the first, second, and third doses of vaccine. For only one RV1 study was the risk incidence presented only for the period after the second dose, owing to the small number of cases (29). The definition of the risk periods varied between studies: 1 to 7 or 3 to 7 days; 8 to 14 or 8 to 21 days; 15 to 21 days after vaccination. In this review, only the results for the risk periods of up to 7 days are reported, since in 5 of the 7 studies that did not include the first 7 days no increased risk was demonstrated $(18,26-29,33,34)$. The following were used as data sources: hospital discharge data (5 studies), data from the Unites States Vaccine Adverse Event Reporting System (VAERS) (2 studies), data from prospective active surveillance programs in hospitals (2 studies), and data from a manufacturer regarding spontaneous reports of intussusception cases after RV5 vaccination (1 study) (Table 1). The studies were carried out in the USA, Australia, Mexico, Singapore, England, and Spain. One study included data from more than one country (USA, Australia, Germany, Japan, Israel). The mean observation period was 4.7 years (median: 5.0 years; range: $1.5-7.5$ years). In 7 of the 10 studies, the intussusception diagnoses in the cases included met the level 1 Brighton criteria (24). Six studies showed a low and 4 a moderate risk of bias (for details see the eTable).

\section{Relative risk of intussusception after RV vaccination}

Figures 1 and 2 show relative risks and pooled estimates from the meta-analyses for the RV1 and RV5 studies and for all studies together. The RR 1 to 7 days after the first dose of vaccine (Figure 1) ranges from 5.3 to 8.5 for RV1 and from 3.5 to 9.9 for RV5, in both cases with low heterogeneity (RV1: $I^{2}=0 \%$; RV5: $I^{2}=$ $30 \%)$. The pooled estimate of the RR after the first dose of vaccine was 5.71 [4.5; 7.25].

The RR 1 to 7 days after the second dose of vaccine (Figure 2) ranges from 1.29 to 3.50 for RV1 and from 1.40 to 2.81 for RV5, with no heterogeneity (RV1: $\mathrm{I}^{2}=$ $0 \%$; RV5: $\mathrm{I}^{2}=0 \%$ ). The pooled estimate of the RR after the second dose of vaccine was 1.69 [1.33; 2.14].

Since only RV5 is administered in the three-dose pattern, the intussusception risk after the third dose of vaccine was studied only in relation to this vaccine. The RR within 1 to 7 days after the third dose of RV5 ranges from 0.75 to 1.74 , with low heterogeneity $\left(\mathrm{I}^{2}=13 \%\right)$ 


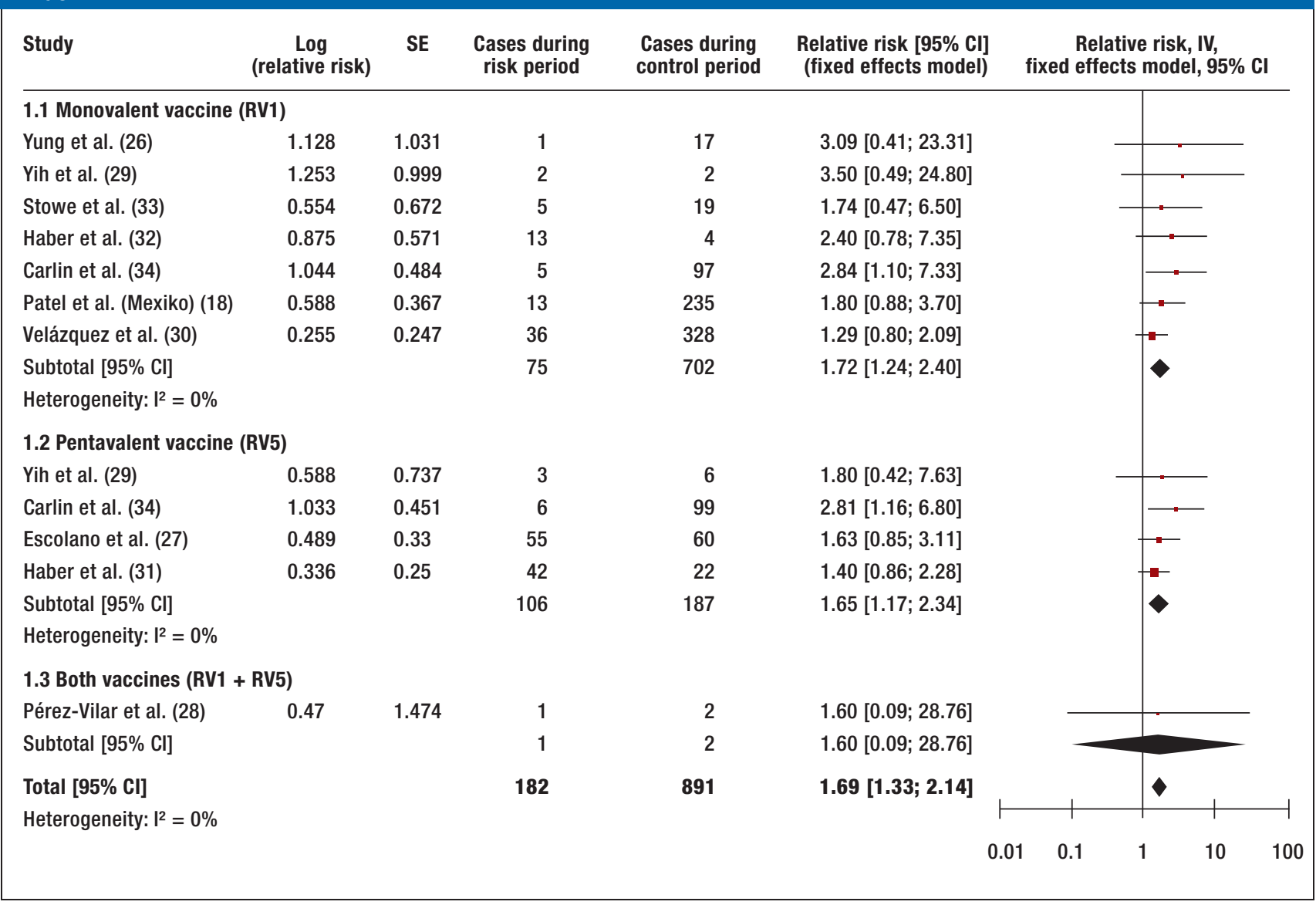

Risk of intussusception within 7 days following the second dose of vaccine

$95 \% \mathrm{Cl}, 95 \%$ confidence interval; IV, inverse variance; SE, standard error

(eFigure 2). The pooled estimate of the RR after the third dose of RV5 vaccine was 1.14 [0.75; 1.74].

The subgroup analysis comparing the data from the SCCS studies with the data from the self-controlled risk interval (SCRI) studies produced very similar results (eFigure 3). The pooled estimate of the RR after the first dose was $5.71[4.47 ; 7.28]$ for the SCCS studies and $4.98[2.89 ; 8.59]$ for the SCRI studies. For the second dose, the pooled estimate was $1.73[1.29 ; 2.31]$ in the SCCS studies and $1.61[1.06 ; 2.44]$ in the SCRI studies.

\section{Assessment of evidence quality}

Using the GRADE method, the quality of the evidence for the intussusception risk after the first dose of vaccine was scored as "high." This was based on the assessment that when the overall potential for bias is low, the evidence quality score can be upgraded two steps, from an initial "low" to "high," in the presence of the criterion "very strong association."

\section{Attributable intussusception risk after $\mathrm{RV}$ vaccination}

The attributable intussusception risk depends on age (Table 2). After the first dose of vaccine, when given at the correct time (age $<3$ months), $1.7[1.1 ; 2.7]$ additional intussusceptions occur per 100000 vaccinated infants, whereas if administration is at the age of $\geq 3$ months, $5.6[4.3 ; 7.2]$ additional intussusceptions occur. In the group aged $<3$ months, $0.25[0.16 ; 0.4]$ additional intussusceptions occur per 100000 vaccinated infants after the second dose, whereas in the $\geq 3$ months group the figure is $0.81[0.63 ; 1.06]$.

\section{Discussion}

The systematic review presented here confirms that the risk of intussusception is increased for 1 to 7 days after RV vaccination, particularly in the period after the first dose of vaccine. If the vaccination takes place at the age of less than 12 weeks, as recommended by STIKO, this means that about every $50000^{\text {th }}$ vaccinated child may suffer intussusception as a consequence of the RV vaccination. If the first vaccination is done later, this becomes every $20000^{\text {th }}$ vaccinated child. The increase in risk after the second vaccination is much lower. Thus, analysis of the recent SCCS studies confirm the risk estimates on which the STIKO recommendation for RV vaccination is based. 
Risk attributable to RV vaccination by age at vaccination

(allowing for the age-specific background incidence of intussusception in Germany)

\begin{tabular}{l|l|l|c} 
& & $<3$ Months & $\geq 3$ Months \\
\hline \multicolumn{2}{l|l}{ Background incidence of intussusception/100 000 infants $^{*}$} & $19.2[12.5 ; 30.4]$ & $61.4[48.0 ; 79.4]$ \\
\hline $\begin{array}{l}\text { Intussusception risk attributable to RV vaccination/100 000 } \\
\text { vaccinated infants }\end{array}$ & First dose & $1.7[1.1 ; 2.7]$ & $5.6[4.3 ; 7.2]$ \\
\cline { 2 - 4 } & Second dose & $0.25[0.16 ; 0.4]$ & $0.81[0.63 ; 1.06]$
\end{tabular}

$95 \%$ confidence intervals in square brackets

*Based on the study by Weiß et al. (8)

Irrespective of which study design was used (SCCS or SCRI), which vaccine was used, and where the study was carried out, the analysis produced very homogeneous results, showing that the risk of intussusception 1 to 7 days after the first dose of vaccine is increased 5.7-fold and the risk after the second dose is increased 1.7 -fold. There is no increase in risk after the third dose of the RV5 vaccine.

At first glance, a RR of 5.7 looks high. When advising parents at the vaccination appointment, however, it is important to emphasize the size of the absolute increase in the individual risk of intussusception that is due to the RV vaccination. Without the $R V$ vaccination, one child in 5208 will have an intussusception during the first 3 months of life, whereas with the RV vaccination the figure is one child in 4785. That is: So long as the vaccination is carried out at the right time, the absolute risk is increased only marginally.

Overall, this risk is markedly lower than the agespecific intussusception risk of children over the age of 3 months without vaccination (one child in 1629). If the first dose of RV vaccine is given at this age, this risk, which is already high even without vaccination, increases markedly and one in every 1493 children suffers an intussusception. This is why it is essential that the first RV vaccination should be given within the first 3 months of life. Based on data from the KV vaccination monitoring program, according to the health insurance company claim data for the 2014 birth cohort, at present still too many children in Germany $(11.2 \%)$ are receiving their first dose of the vaccine at over 3 months of age (Thorsten Rieck, Robert Koch Institute, personal communication) (36).

The hospital discharge data relating to annual cases of intussusception in infants show (eFigure 4) that, with birth cohort size remaining almost steady, the number of cases of intussusception has not risen either since vaccines were licensed in 2006 or since the STIKO vaccination recommendation came into effect in 2013, even though RV vaccination rates have been continuously rising since 2006 (37). This demonstrates that the increase in intussusception risk during a short time window after vaccination has had no measurable effect on the incidence of intussusception in children in Germany. Against this there is the benefit of RV vacci- nation. Impact analysis has shown a statistically significant drop in the proportion of RV-related hospital admissions, by about $25 \%$ to $36 \%$, owing to vaccination (37).

The present systematic review is the first to analyze all the current published data from SCCS studies - the study design best suited for this purpose - on the two vaccines available at present. The very homogeneous study estimates, the robustness of the pooled estimates in subgroup analyses, and the presence of a negative dose-effect relationship support the assessment that the evidence used is of high quality.

\section{Limitations}

The calculation of attributable risk (AR) was carried out by extrapolating the results from the SCCS studies to the cohort setting of the German population. This could have introduced bias into the AR, but the extent and direction of this error cannot be estimated.

\section{Conclusion}

The vaccinating physician should start the series of vaccinations at the right time, when the child is between the ages of 6 and 12 weeks - as recommended by STIKO - and should explain to the parents that the $\mathrm{RV}$ vaccination is associated with a slightly increased risk of intussusception. Intussusception is an intestinal emergency and is the commonest cause of acute abdomen in infants. Children with intussusception ileus require immediate diagnosis and treatment. Treatment, which is usually conservative, is important, as rapid action is associated with a high chance of success $(80 \%$ to $90 \%$ ) and a very good prognosis; a fatal outcome is the exception (38). If the infant develops symptoms that might indicate intussusception during the 7 days after vaccination (e.g., colicky abdominal pain, bilious vomiting, "currant jelly" stool), immediate medical attention is required.

\section{Conflict of interest statement}

The authors declare that no conflict of interest exists.

Manuscript received on 18 July 2016, revised version accepted on 3 January 2017

Translated from the original German by Kersti Wagstaff, MA. 


\section{KEY MESSAGES}

- Before vaccination was introduced in Germany, gastroenteritis caused by rotaviruses was the commonest notifiable disease in children under the age of 5 . The German Standing Committee on Vaccination recommended RV vaccination for infants in 2013, pointing out that a slightly increased risk of intussusception would occur.

- This meta-analysis has confirmed that the risk of intussusception is increased during the 7 days following each of the first two doses of RV vaccine. The pooled estimate of relative risk is 5.71 after the first dose and 1.69 after the second dose.

- The risk attributable to RV vaccination depends on age. In children vaccinated at less than 3 months of age, 1 to 3 additional intussusceptions per 100000 are expected; in those vaccinated later than the recommended age, 4 to 8 additional intussusceptions per 100000 are expected.

- The absolute risk of intussusception is only marginally increased by vaccination carried out at the right time. Without vaccination, one in 5208 children will suffer an intussusception. With vaccination, the figure is one in 4785.

- Vaccination should be carried out within the recommended time window in order to keep the risk of intussusception as low as possible.

\section{REFERENCES}

1. Parashar UD, Gibson CJ, Bresee JS, Glass RI: Rotavirus and severe childhood diarrhea. Emerg Infect Dis 2006; 12: 304-6.

2. Tate JE, Burton AH, Boschi-Pinto C, Steele AD, Duque J, Parashar UD: 2008 estimate of worldwide rotavirus-associated mortality in children younger than 5 years before the introduction of universal rotavirus vaccination programmes: a systematic review and metaanalysis. Lancet Infect Dis 2012; 12: 136-41.

3. Koch J, Wiese-Posselt M: Epidemiology of rotavirus infections in children less than 5 years of age: Germany, 2001-2008. Pediatr Infect Dis J 2011; 30: 112-7.

4. Rieck T, Feig M, Eckmanns T, et al.: Vaccination coverage among children in Germany estimated by analysis of health insurance claims data. Hum Vaccin Immunother; 10: 476-84.

5. European Medicines Agency: Rotarix. Scientific discussion, 11 September 2006. www.ema.europa.eu/docs/en_GB/document library/ EPAR_-_Scientific_Discussion/human/000639/WC500054589.pdf (last accessed on 30 March 2012).

6. European Medicines Agency: RotaTeq. Scientific discussion, 21 September 2006. www.ema.europa.eu/docs/en_GB/document_ library/EPAR_-_Scientific_Discussion/human/000669/WC 500054183.pdf (last accessed on 9 January 2017).

7. Bines JE, Liem NT, Justice FA, et al.: Risk factors for intussusception in infants in Vietnam and Australia: adenovirus implicated, but not rotavirus. J Pediatr 2006; 149: 452-60.

8. Weiß S, Streng A, Kries RV, Liese J, Wirth S, Jenke AC: Inzidenz der Invagination bei Säuglingen in Deutschland. Eine Schätzung anhand der Capture-Recapture-Methode. Klinische Pädiatrie 2011; 223: 419-23.

9. Kramarz P, France EK, Destefano F, et al.: Population-based study of rotavirus vaccination and intussusception. Pediatr Infect Dis $J$ 2001; 20: 410-6.
10. Centers for Disease Control and Prevention (CDC): Intussusception among recipients of rotavirus vaccine, United States, 1998-1999. Morb Mortal Wkly Rep (MMWR) 1999; 48: 577-81.

11. Ruiz-Palacios GM, Perez-Schael I, Velazquez FR, et al.: Safety and efficacy of an attenuated vaccine against severe rotavirus gastroenteritis. N Engl J Med 2006; 354: 11-22.

12. Vesikari T, Matson DO, Dennehy P, et al.: Safety and efficacy of a pentavalent human-bovine (WC3) reassortant rotavirus vaccine. N Engl J Med 2006; 354: 23-33.

13. Belongia EA, Irving SA, Shui IM, et al.: Real-time surveillance to assess risk of intussusception and other adverse events after pentavalent, bovine-derived rotavirus vaccine. Pediatr Infect Dis J 2010; 29: 1-5.

14. Shui IM, Baggs J, Patel M, et al.: Risk of intussusception following administration of a pentavalent rotavirus vaccine in US infants. JAMA 2012; 307: 598-604.

15. Centers for Disease Control and Prevention (CDC): Postmarketing monitoring of intussusception after RotaTeq (TM) vaccination, United States, February 1, 2006 - February 15, 2007. Morb Mortal Wkly Rep (MMWR) 2007; 56: 218-22.

16. Loughlin J, Mast TC, Doherty MC, Wang FT, Wong J, Seeger JD: Postmarketing evaluation of the short-term safety of the pentavalent rotavirus vaccine. Pediatr Infect Dis J 2012; 31: 292-6.

17. Haber P, Patel M, Izurieta HS, et al.: Postlicensure monitoring of intussusception after RotaTeq vaccination in the United States, February 1, 2006, to September 25, 2007. Pediatrics 2008; 121 : 1206-12.

18. Patel MM, López-Collada VR, Bulhões MM, et al.: Intussusception risk and health benefits of rotavirus vaccination in Mexico and Brazil. N Engl J Med 2011; 364: 2283-92.

19. Buttery JP, Danchin MH, Lee KJ, et al.: Intussusception following rotavirus vaccine administration: post-marketing surveillance in the National Immunization Program in Australia. Vaccine 2011; 29: 3061-6.

20. Australian Government, Department of Health and Ageing, Therapeutic Goods Administration: Rotavirus vaccination and risk of intussusception. www.tga.gov.au/safety/alerts-medicine-rotavi rus-110225.htm (last accessed on 8 February 2017).

21. Escolano S, Farrington CP, Hill C, Tubert-Bitter P: Intussusception after rotavirus vaccination-spontaneous reports. N Engl J Med 2011; 365: 2139.

22. Oberle D, Jenke AC, von Kries R, Keller-Stanislawski B: [Rotavirus vaccination: a risk factor for intussusception?] Bundesgesundheitsblatt - Gesundheitsforschung - Gesundheitsschutz 2014; 57 : 234-41.

23. Moher D, Liberati A, Tetzlaff J, Altman DG: Preferred reporting items for systematic reviews and meta-analyses: the PRISMA statement. BMJ 2009; 339: b2535.

24. Bines JE, Kohl KS, Forster J, et al.: Acute intussusception in infants and children as an adverse event following immunization: case definition and guidelines of data collection, analysis, and presentation. Vaccine 2004; 22: 569-74.

25. Weldeselassie YG, Whitaker HJ, Farrington CP: Use of the selfcontrolled case-series method in vaccine safety studies: review and recommendations for best practice. Epidemiol Infect 2011; 139: 1805-17.

26. Yung CF, Chan SP, Soh S, Tan A, Thoon KC: Intussusception and monovalent rotavirus vaccination in singapore: selfcontrolled case series and risk-benefit study. J Pediatr 2015; 167: 163-8.

27. Escolano S, Hill C, Tubert-Bitter P: Intussusception risk after RotaTeq vaccination: evaluation from worldwide spontaneous reporting data using a self-controlled case series approach. Vaccine 2015; 33: 1017-20.

28. Pérez-Vilar S, Díez-Domingo J, Puig-Barberà J, Gil-Prieto R, Romio S: Intussusception following rotavirus vaccination in the Valencia region, Spain. Hum Vaccin Immunother 2015; 11: 1848-52. 
29. Yih WK, Lieu TA, Kulldorff M, et al.: Intussusception risk after rotavirus vaccination in U.S. infants. New Engl J Med 2014; 370: 503-12.

30. Velázquez FR, Colindres RE, Grajales C, et al.: Postmarketing surveillance of intussusception following mass introduction of the attenuated human rotavirus vaccine in Mexico. Pediatr Infect Dis $J$ 2012; 31: 736-44

31. Haber P, Patel M, Pan Y, et al.: Intussusception after rotavirus vaccines reported to US VAERS, 2006-2012. Pediatrics 2013; 131: 1042-9.

32. Haber P, Parashar UD, Haber M, DeStefano F: Intussusception after monovalent rotavirus vaccine-United States, Vaccine Adverse Event Reporting System (VAERS), 2008-2014. Vaccine 2015; 33: 4873-7

33. Stowe J, Andrews N, Ladhani S, Miller E: The risk of intussusception following monovalent rotavirus vaccination in England: a self-controlled case-series evaluation. Vaccine 2016; 34: 3684-9.

34. Carlin JB, Macartney KK, Lee KJ, et al.: Intussusception risk and disease prevention associated with rotavirus vaccines in Australia's National Immunization Program. CID 2013; 57: 1427-34.

35. Ciarlet M, Sani-Grosso R, Yuan G, et al.: Concomitant use of the ora pentavalent human-bovine reassortant rotavirus vaccine and oral poliovirus vaccine. Pediatr Infect Dis J 2008; 27: 874-80.
36. Rieck T, Feig M, Wichmann 0, Siedler A: Aktuelles aus der KVImpfsurveillance - Impfquoten der Rotavirus-, Masern-, HPV- und Influenza-Impfung in Deutschland. Epid Bull 2017; 1: 1-12.

37. Dudareva-Vizule S, Koch J, An der Heiden M, Oberle D, KellerStanislawski B, Wichmann 0: Impact of rotavirus vaccination in regions with low and moderate vaccine uptake in Germany. Hum Vaccin Immunother 2012; 8: 1407-15

38. Gfroerer S, Fiegel H, Rolle U: Invagination. Monatsschrift Kinderheilkunde 2009; 157: 917-24.

\section{Corresponding author:}

Dr. med. Judith Koch

Robert Koch-Institut, Impfprävention

Seestr. 10, 13353 Berlin, Germany

KochJ@rki.de

\section{Supplementary material}

For eReferences please refer to:

www.aerzteblatt-international.de/ref1517

eFigures, eBoxes, eTable:

www.aerzteblatt-international.de/17m0255

\section{CLINICAL SNAPSHOT}

\section{Sawtooth Waves-Atrial Flutter in a Premature Infant}

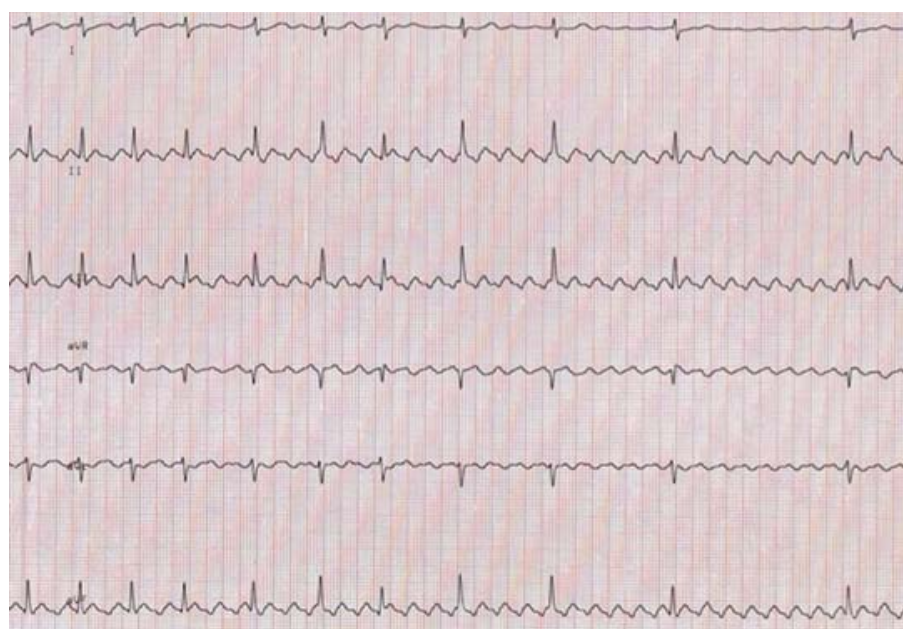

An infant was delivered prematurely by cesarean section in gestational week $34+3$ because of intermittent intrauterine tachycardia (heart rate ca. 190-200/min). Its weight at birth was $2795 \mathrm{~g}$. The postnatal Apgar-score (7/8/9), complete blood count, and clinical chemistry values were normal. 15 minutes after birth, the newborn infant developed cardiopulmonary instability and was intubated and given intravenous catecholamines for cardiovascular support. Echocardiography revealed hypertrophic cardiomyopathy of the left ventricle and dilatation of the hepatic veins and inferior vena cava. An EcG aroused the suspicion of atrial flutter. This was unmasked by the administration of adenosine (with CPR immediately available if necessary), and a persistent sinus rhythm was then successfully re-established with cardioversion (2 joules). The abnormal findings on echocardiography, which were due to the abnormal rhythm, resolved thereafter. No further drugs were given, and atrial flutter did not recur. Fetal cardiac arrhythmias arise in only about $2 \%$ of pregnancies, and atrial flutter makes up at most $3 \%$ of these arrhythmias. It is thus a rare entity among neonates, and among premature neonates in particular.

Dr. med. Martin Poryo, Dr. med. Mojtaba Abedini, Dr. med. Günther Löffler, Klinik für Pädiatrie, Universitätsklinikum des Saarlandes, Homburg/Saar, martin.poryo@uks.eu

\section{Conflict of interest statement}

The authors state that they have no conflict interest

Translated from the original German by Ethan Taub, M.D.

\section{Cite this as:}

Poryo M, Abedini M, Löffler G: Sawtooth Waves_atrial flutter in a premature infant. Dtsch Arztebl Int 2017; 114: 255. D0l: 10.3238/arztebl.2017.0255 


\section{Supplementary material to:}

\section{Risk of Intussusception After Rotavirus Vaccination}

A Systematic Literature Review and Meta-Analysis

by Judith Koch, Thomas Harder, Rüdiger von Kries, and Ole Wichmann

Dtsch Arztebl Int 2017; 114: 255-62. D0l: 10.3238/arztebl.2017.0255

\section{EREFERENCES}

e1. Guyatt G, Oxman AD, Akl EA, et al.: GRADE guidelines: 1. Introduction-GRADE evidence profiles and summary of findings tables. J Clinical Epidemiol 2011; 64: 383-94.

e2. Wood N, Cannings K, Quinn H, et al.: Rotavirus vaccines and intussusception: An increased risk. J Paediatr Child Health 2011; 47 (Suppl 2): 16.

e3. Haber P, Patel M, Pan Y, et al.: Monitoring of intussusception after rotavirus vaccines-united states, vaccine adverse event reporting system (VAERS), 2006-2012. Abstracts of the 29th International Conference on Pharmacoepidemiology \& Therapeutic Risk Management. August 25-28, 2013. Montreal, Canada. Pharmacoepidemiol Drug Saf 2013; 22 (Suppl 1): 206.

e4. Escolano S, Hill C, Tubert-Bitter P: A new self-controlled case series method for analyzing spontaneous reports of adverse events after vaccination. Am J Epidemiol 2013; 178(9): 1496-504.

e5. Velazquez FR, Colindres R, Grajales C, et al.: Postmarketing surveillance of intussusception following mass introduction of the human rotavirus vaccine in Mexico: An interim analysis. Acta Paediatr 2010; 99: 92.

e6. Leino T, Ollgren J, Stromberg N, Elonsalo U: Evaluation of the intussusception risk after pentavalent rotavirus vaccination in Finnish infants. PloS ONE 2016; 11: e0144812.

e7. Quinn HE, Wood NJ, Cannings KL, et al.: Intussusception after monovalent human rotavirus vaccine in Australia: severity and comparison of using healthcare database records versus case confirmation to assess risk. Pediatric Infect Dis J 2014; 33: 959-65. 


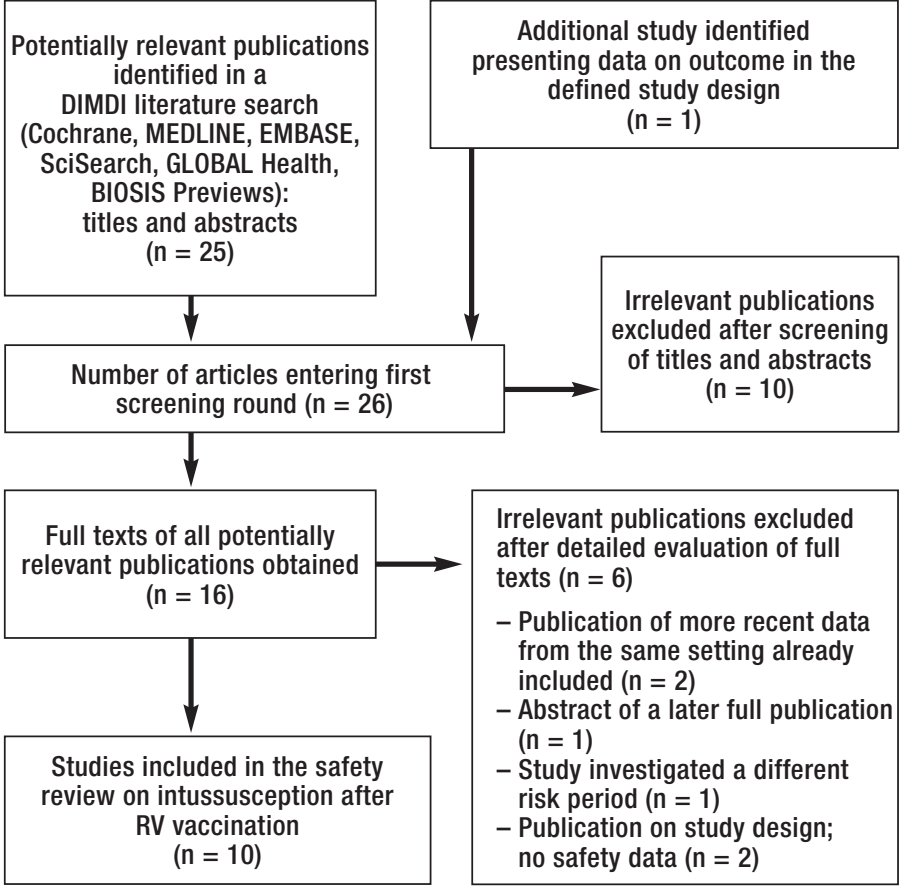

Flow diagram showing literature search method

DIMDI, German Institute of Medical Documentation and Information (Deutsches Institut für Medizinische Dokumentation und Information); RV, rotavirus 


\section{eFIGURE 2}

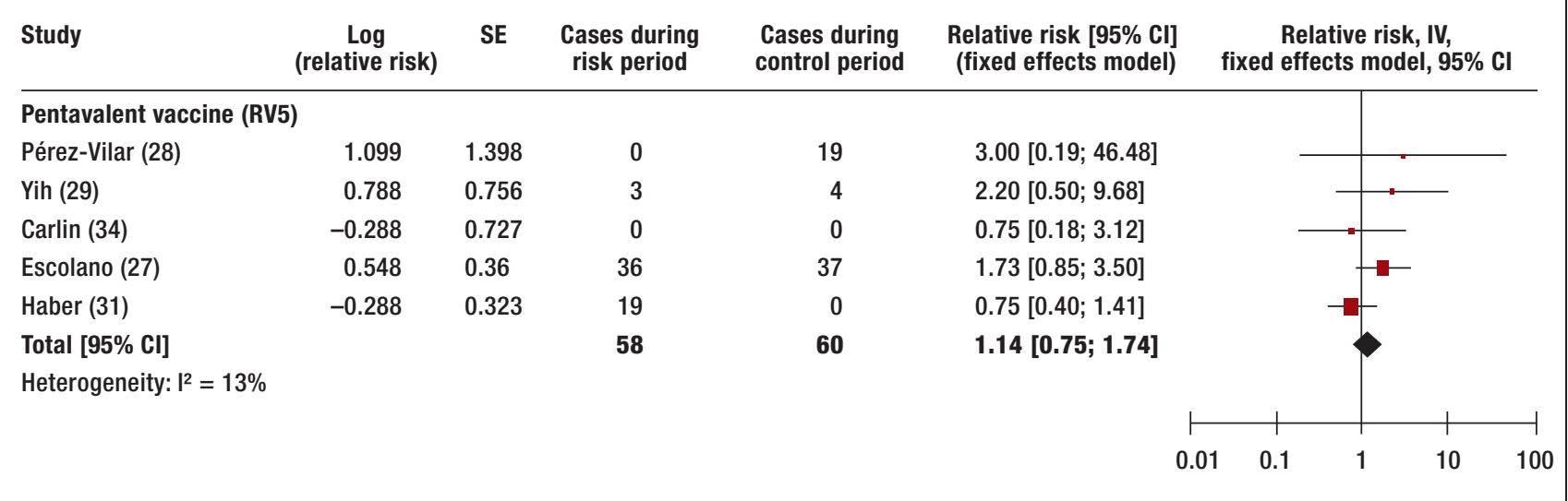

Risk of intussusception within 7 days following the third dose of vaccine

$95 \% \mathrm{Cl}, 95 \%$ confidence interval; IV, inverse variance; SE, standard error 
eFIGURE 3

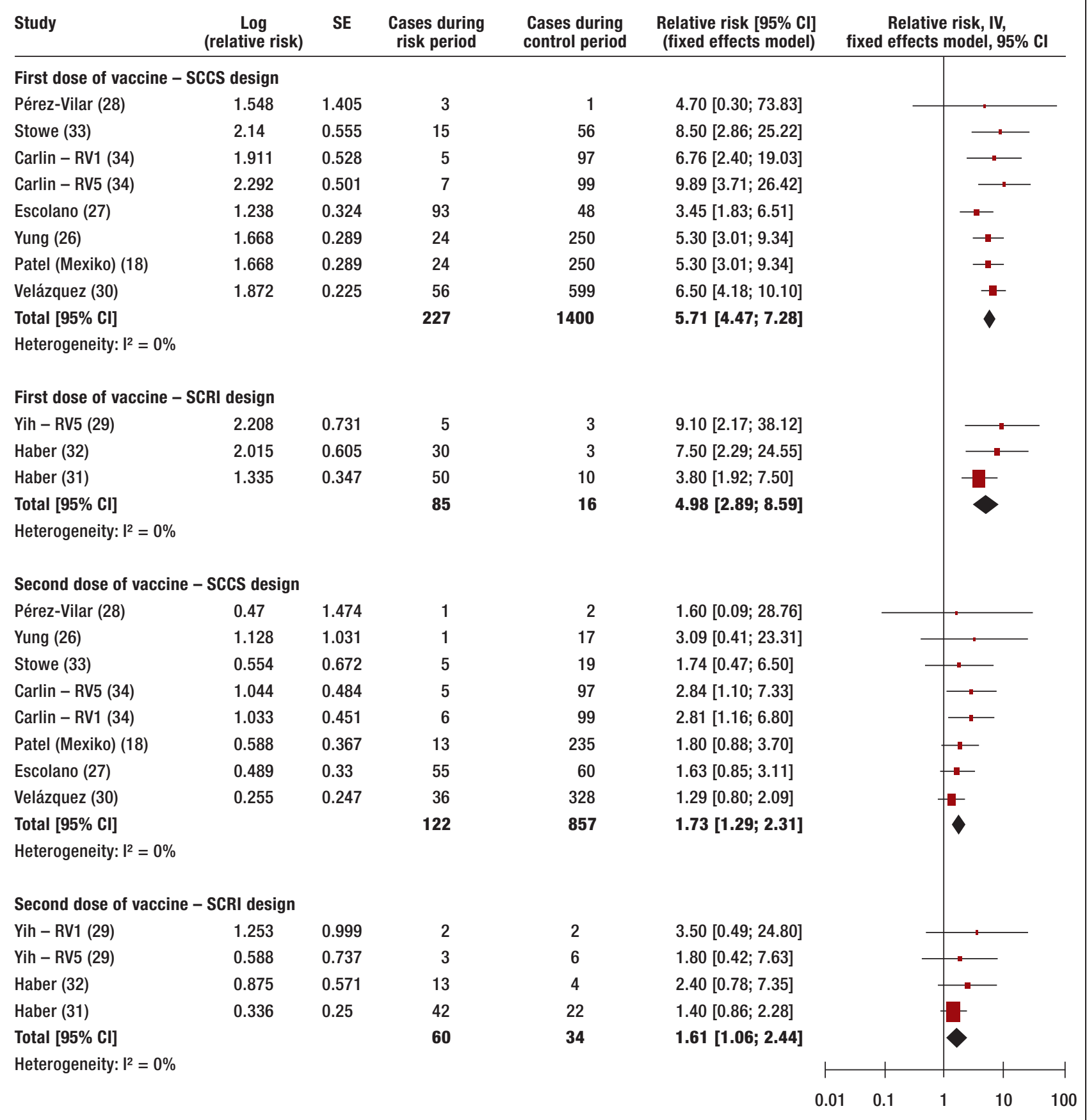

Risk of intussusception within 7 days following the first and second doses of vaccine — comparison of SCCS design and SCRI design studies $95 \% \mathrm{Cl}, 95 \%$ confidence interval; IV, inverse variance; RV, rotaviruses; RV1, monovalent vaccine; RV5, pentavalent vaccine; SCCS, self-controlled case series; SCRI, self-controlled risk interval; SE standard error 


\section{eFIGURE 4}

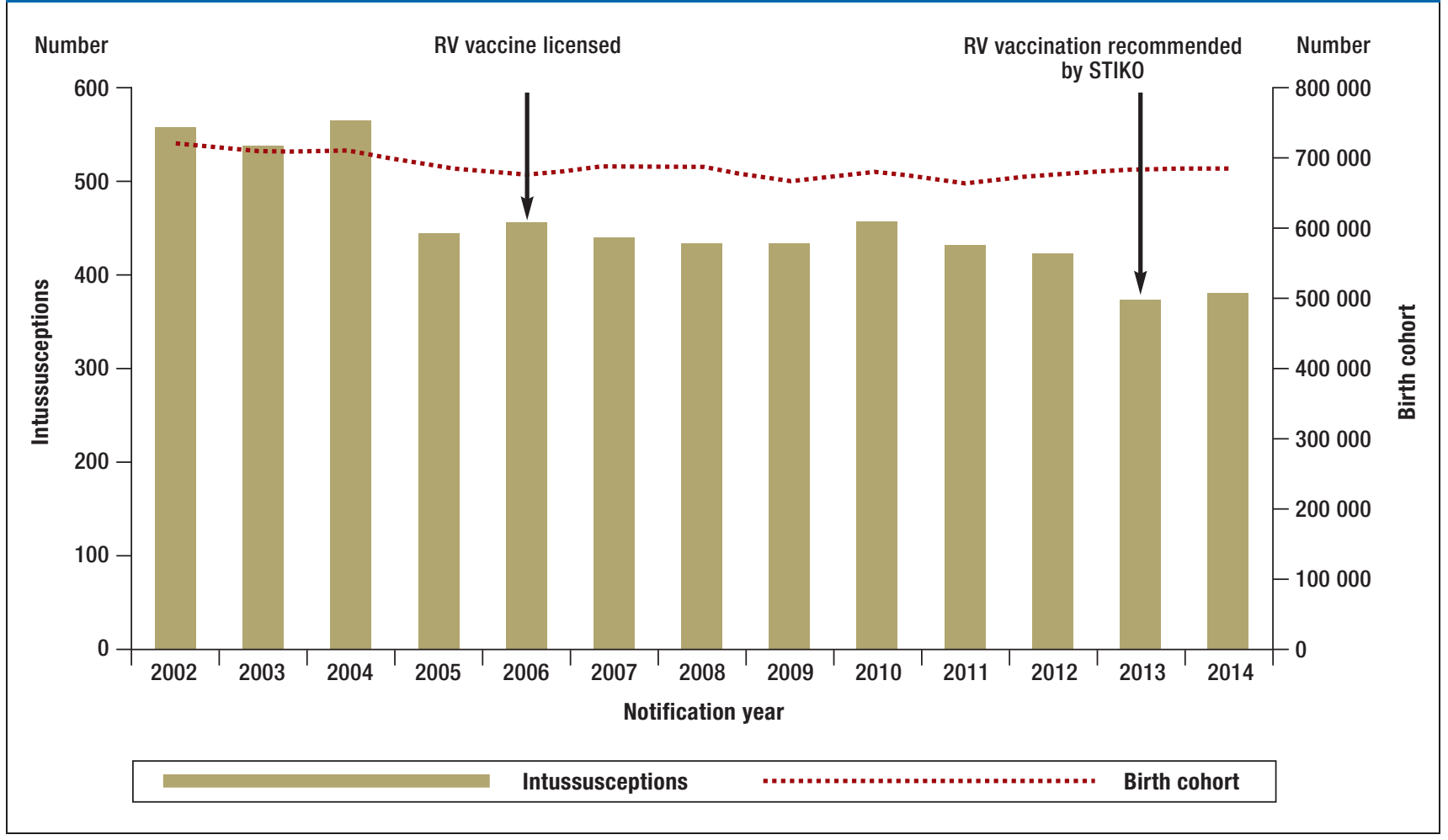

Number of intussusceptions in infants aged $<1$ year; hospital discharge diagnoses taken from Federal Stastistical 0ffice health reports, 2002-2014 RV, rotavirus; STIKO, German Standing Committee on Vaccination (Ständige Impfkommission) 


\section{Details of study design}

The self-controlled case series (SCCS) method is a so-called "conditional cohort design," which combines features of the cohort study and those of the case-control study. The object of analysis is a cohort of study participants, all of whom show the event in question (in this case, intussusception). What is investigated is the risk that the event of interest will occur during a specified period after the vaccination ("the risk period") in comparison to the risk that it will occur during another time period ("the control period"). In the studies included in this analysis, the risk period was defined as the period of up to 7 days after vaccination; the control period was defined as the period before the vaccination or more than 21 days after it. The study estimate (relative risk) is calculated from the relationship between the risk that an intussusception will occur during the risk period and the risk of intussusception during the control period, using conditional Poisson regression. Because only "cases" are analyzed, which de facto function as their own "controls," this study design in itself adjusts for time-independent confounding factors. This review and meta-analysis also included studies using the self-controlled risk interval (SCRI) design, a simplified version of the SCCS design. In the SCRI design, usually a shorter period is regarded as the control period, and all study participants receive the vaccination. 


\section{Supplement regarding methods}

\section{Search strategy}

MEDLINE, EMBASE, Cochrane Central Register of Controlled Trials, SciSearch, GLOBAL Health, and BIOSIS Previews searched (search date: 10 July 2015; search updated: 4 July 2016):

\#1 rotavirus

\#2 intussusception

\#3 \#1 AND \#2

$\# 4$ self controlled case series

\#5 self controlled risk

\#6 \#4 OR \#5

\#7 \#3 AND \#6

In addition, the reference lists of the studies included and the review articles identified were screened for further studies. No restrictions as to publication status or language were imposed.

\section{Data extraction}

From original articles that fulfilled the inclusion criteria, two investigators ( $\mathrm{JK}, \mathrm{TH})$ working independently of each other extracted study characteristics and data for the various doses of vaccine (number of observed cases during the risk and control periods, risk incidences ([RI: incidence during the risk period divided by incidence during the control period]).

\section{- 3. Determination of risk of bias and assessment of evidence quality}

To determine the risk of bias in the studies included in this review, the Cochrane Risk of Bias Assessment Tool for NonRandomized Studies of Interventions (ACROBAT-NRSI) was employed (www.riskofbias.info). Discrepancies between the investigators were discussed until a consensus was reached. The quality of the evidence was assessed using the GRADE (Grading of Recommendations Assessment, Development and Evaluation) criteria (e1).

\section{Data synthesis}

From the extracted data, relative risks (RR) and the corresponding 95\% confidence intervals were calculated or taken directly from the respective publications. The two vaccines were analyzed both separately and together. When more than one study was available, a meta-analysis was carried out. A subgroup analysis of RR during the 1 to 7 days after the first and second vaccine doses was carried out, comparing the data from the self-controlled case series (SCCS) with those from the selfcontrolled risk interval (SCRI) studies, the latter being a simplified version of the SCCS study design with a shorter time period as the control period. If heterogeneity was shown (determined on the basis of the $1^{2}$ statistic), a random effects model was used; otherwise, the data were summarized using a fixed effects model. In accordance with the recommendations of the Cochrane Collaboration, since fewer than 10 studies were available for analysis no test for publication bias was carried out. All calculations were performed using STATA 14 software (StatCorp, College Station, Texas, USA) and Review Manager 5 (Cochrane Collaboration, The Nordic Cochrane Centre).

- 5. Publications that were not included after full text screening (e2-e7). 


\section{eTABLE}

Risk of bias in the studies identified in the systematic review and included in the meta-analysis

\begin{tabular}{|c|c|c|c|c|c|c|c|c|}
\hline Study & $\begin{array}{l}\text { Confounding } \\
\text { factors }\end{array}$ & Selection bias & \begin{tabular}{|c|} 
Documentation \\
of the \\
intervention
\end{tabular} & $\begin{array}{c}\text { Variant } \\
\text { intervention }\end{array}$ & Missing data & $\begin{array}{l}\text { Documentation } \\
\text { of the outcome }\end{array}$ & $\begin{array}{l}\text { Publication } \\
\text { bias }\end{array}$ & $\begin{array}{l}\text { Overall risk o } \\
\text { bias }\end{array}$ \\
\hline Yung et al. (26) & ++ & ++ & ++ & ++ & ++ & ++ & ++ & ++ \\
\hline Escolano et al. (27) & ++ & + & ++ & ++ & ++ & $?$ & ++ & ++ \\
\hline Carlin et al. (34) & ++ & ++ & ++ & ++ & ++ & ++ & ++ & ++ \\
\hline $\begin{array}{l}\text { Pérez-Vilar et al. } \\
\text { (28) }\end{array}$ & + * & ++ & ++ & ++ & ++ & ++ & ++ & + \\
\hline Yih et al. (29) & $+*$ & ++ & ++ & ++ & ++ & ++ & ++ & + \\
\hline $\begin{array}{l}\text { Velázquez et al. } \\
\text { (30) }\end{array}$ & ++ & ++ & ++ & ++ & ++ & ++ & ++ & ++ \\
\hline Patel et al. (18) & ++ & ++ & ++ & ++ & ++ & ++ & ++ & ++ \\
\hline Haber et al. (31) & $+*$ & ++ & ++ & ++ & ++ & ++ & ++ & + \\
\hline Haber et al. (32) & $+*$ & ++ & ++ & ++ & ++ & ++ & ++ & + \\
\hline Stowe et al. (33) & ++ & ++ & ++ & ++ & ++ & ++ & ++ & ++ \\
\hline
\end{tabular}

++ low risk; + moderate risk; ? no information;

* only vaccinated infants, not adjusted 